\title{
Hormonal changes associated with experimentally produced cystic ovaries in the cow
}

\author{
R. Nadaraja* and W. Hansel† \\ Department of Animal Sciences, Cornell University, Ithaca, New York 14853, U.S.A.
}

\begin{abstract}
Summary. Cystic ovaries were experimentally produced by i.m. injection of $5 \mathrm{mg}$ oestradiol valerate on Day 16 of the oestrous cycle or by s.c. injection of $100 \mathrm{ml}$ antiserum to bovine LH before the onset of oestrus. The length of the cycle after both treatments was altered. The cysts formed after oestradiol injection were relatively small ( $2 \cdot 5-3 \mathrm{~cm}$ diameter) and thin-walled, and were accompanied by relatively low plasma oestrone and high oestradiol levels; those after $\mathrm{LH}$ antiserum treatment were large $(5 \cdot 0-6 \cdot 0 \mathrm{~cm})$ and firm and associated with relatively low oestradiol and high oestrone levels. The results of the hormone assays after treatment support the theory that cystic ovaries could result from premature LH release or an insufficiency of $\mathrm{LH}$ at the time of ovulation.
\end{abstract}

\section{Introduction}

Estimations of the incidence of cystic ovaries in cattle vary but the condition is considered to be one cause of infertility. The development of cystic ovaries has been related to uterine infection (Garm, 1949), high milk production (Casida \& Chapman, 1951), seasonal changes (Roberts, 1971), hereditary predisposition (Henricson, 1957), and pituitary dysfunction (Yamauchi, Ashida \& Inui, 1954; McEntee, 1958; Donaldson \& Hansel, 1968). Exogenous oestrogens and other oestrogenic substances (e.g. stilboestrol) are known to produce cystic ovaries. Wiltbank (1966) observed that a single injection of $5 \mathrm{mg}$ oestradiol valerate on the 15th or 16th day of the oestrous cycle produced large cystic ovaries in 7 out of 10 heifers. Schechter (1973) studied the use of an antiserum to bovine $\mathrm{LH}$ for control of the oestrous cycle and observed cystic ovaries in some of the heifers treated late in the cycle. These results suggest that cystic ovaries in cattle may result from premature release of LH or from the release of amounts of $\mathrm{LH}$ inadequate to cause ovulation. The present study was therefore designed to test the hypothesis that the release of $\mathrm{LH}$ at an inappropriate time in follicular development or an insufficiency of LH at the normal time of ovulation could cause cystic ovaries.

\section{Materials and Methods}

Two experiments were carried out during the months of May, June and July. In both cystic development of the ovary was diagnosed by rectal palpation in cows which had no corpus luteum, but had a follicular structure $2.5 \mathrm{~cm}$ or more in diameter which was retained in the same location for at least 10 days.

\section{Experiment 1}

Five Holstein heifers 20-36 months of age and having histories of normal oestrous cycles were used. Oestradiol valerate $(5 \mathrm{mg}$ ) in sesame oil was administered intramuscularly (i.m.) on Day 16 (oestrus $=$ Day 0 ) of the cycle. A pretreatment blood sample of $50 \mathrm{ml}$ was taken from the jugular vein, thereafter daily blood samples $(50 \mathrm{ml})$ were taken for 21 days at 08.00 hours. The blood samples

\footnotetext{
* Present address: National Council for Scientific Research, Animal Productivity Research Unit, P. O. Box 49, Chilanga, Lusaka, Zambia.

$\uparrow$ Reprint requests to Dr. W. Hansel.
} 
were centrifuged at $4^{\circ} \mathrm{C}$ within $30 \mathrm{~min}$ of collection, and aliquots were stored at $-10^{\circ} \mathrm{C}$ until analysis for progesterone, $\mathrm{LH}$, oestrone and oestradiol-17ß. The animals were observed for oestrus twice daily from the beginning of the experiment for about 2 months. The ovaries and the reproductive tract were examined per rectum every 3 days for the period of blood sampling and clinical notes were maintained.

\section{Experiment 2}

Six Holstein heifers 20-36 months of age with histories of normal oestrous cycles were used. The animals were closely observed for oestrus three times a day beginning several days before the expected oestrus. When the animals began to show the first signs of oestrus (restlessness, presence of vaginal mucus and mounting other animals), they were examined rectally and if judged as coming into oestrus were injected s.c. with $100 \mathrm{ml}$ antiserum to bovine LH (Snook, Brunner, Saatman \& Hansel, 1969). A pretreatment blood sample of $50 \mathrm{ml}$ was taken from the jugular vein, and daily blood samples of $50 \mathrm{ml}$ were then taken at 08.00 hours for 21 days. The blood samples were centrifuged, divided into aliquots, stored and assayed as described below, except that the LH assay was modified as described by Schechter (1973) to overcome the interference of the excess circulating antibodies. Clinical examinations were made and notes maintained as in Exp. 1. This antiserum had a high potency: $0.1 \mathrm{ml}$ serum neutralized $80 \mu \mathrm{g}$ bovine $\mathrm{LH}$ in the rat ventral prostate weight assay.

\section{Hormone assays}

Progesterone. The radioimmunoassay used was that described by Hixon, Nadaraja, Schechter \& Hansel (1973). The coefficient of variation between assays was $4.7 \%$.

Oestrogen. The radioimmunoassay method of Echternkamp \& Hansel (1973) was used for oestrone determinations, and for oestradiol-17 $\beta$ except that for the latter a specific oestradiol antibody was employed. The coefficients of variation between assays were $16.36 \%$ for oestrone and $14.4 \%$ for oestradiol-17ß.

$L H$. The solid-phase radioimmunoassay described by Hobson \& Hansel (1972) was used. The coefficient of variation between assays was $6.8 \%$.

\section{Results}

\section{Experiment 1}

Oestrus. All 5 heifers came into oestrus within $24-48 \mathrm{hr}$ after oestradiol valerate treatment. In addition, four of them exhibited a second oestrus $48-120 \mathrm{hr}$ after the first. Oestrus was subsequently delayed in all the heifers and none returned to oestrus earlier than $\mathbf{4 0}$ days after the last oestrus. Untreated control heifers housed and fed in the same way as the treated animals continued to cycle normally during this period.

Cyst formation. Rectal examinations showed that cysts formed in the ovaries of all five treated heifers from follicles which developed after the induced oestrus. All of the cysts were between 2.5 and $3.0 \mathrm{~cm}$ in diameter and appeared to be thin-walled.

Hormonal responses. These are shown in Table 1. Elevated concentrations of plasma oestradiol were noted in all animals within 24 hours after treatment. Elevated plasma LH concentrations preceded, or were associated with, the plasma oestradiol peaks in 3 animals and the plasma oestrone concentrations remained lower than plasma oestradiol concentrations. Corpora lutea were not detected after the induced oestrus and plasma progesterone concentrations remained low. After the initial peak, concentrations of oestradiol declined to values within the normal range in Heifers 1 , 2 and 5 and remained relatively low. Plasma levels in Heifers 3 and 4 remained high. Heifer 4 became nymphomaniacal, exhibiting frequent, irregular or continuous signs of oestrus, nervousness, restlessness, continual mounting of other cows, and relaxation of the pelvic ligaments and the cycle length 


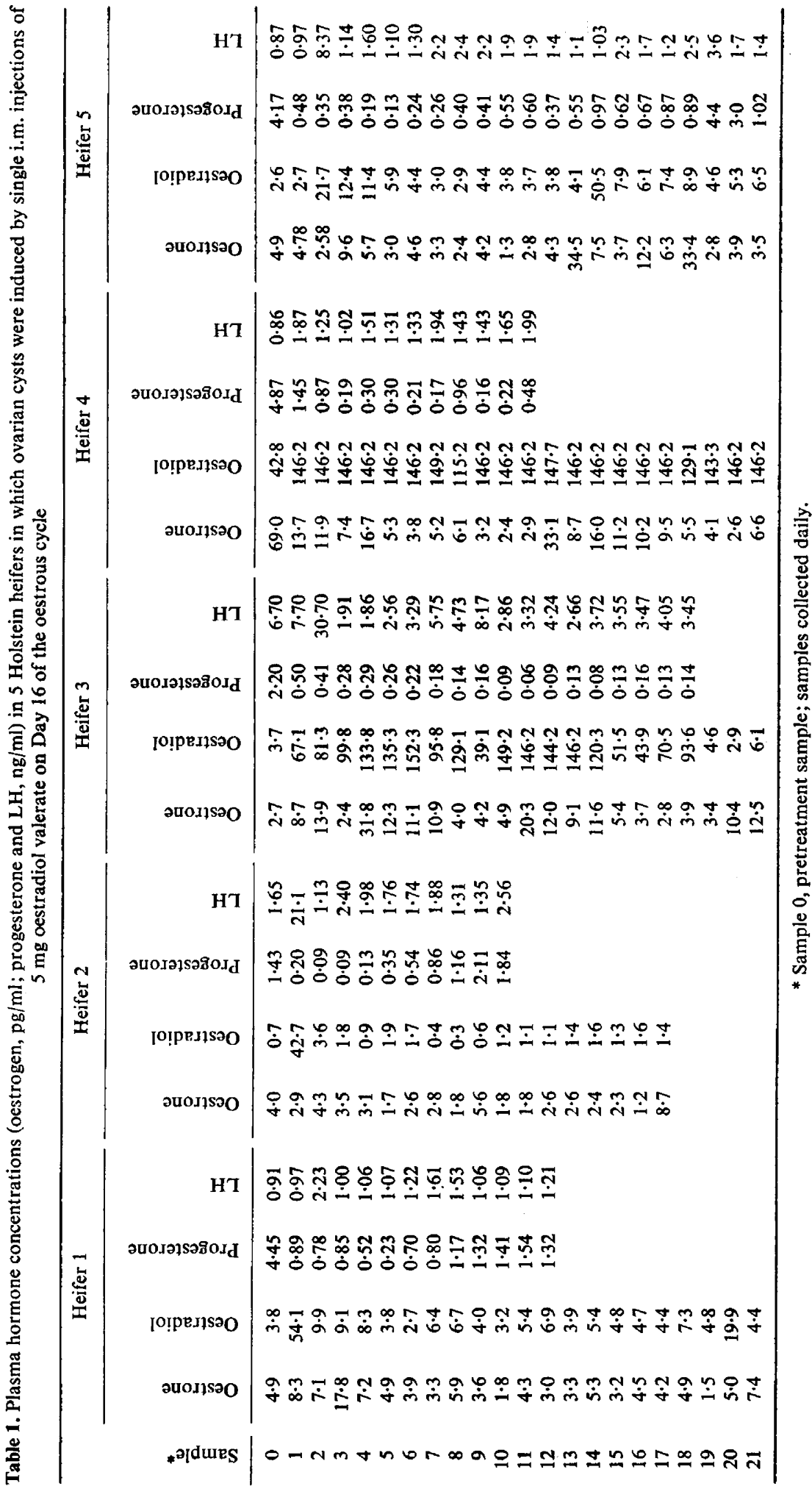




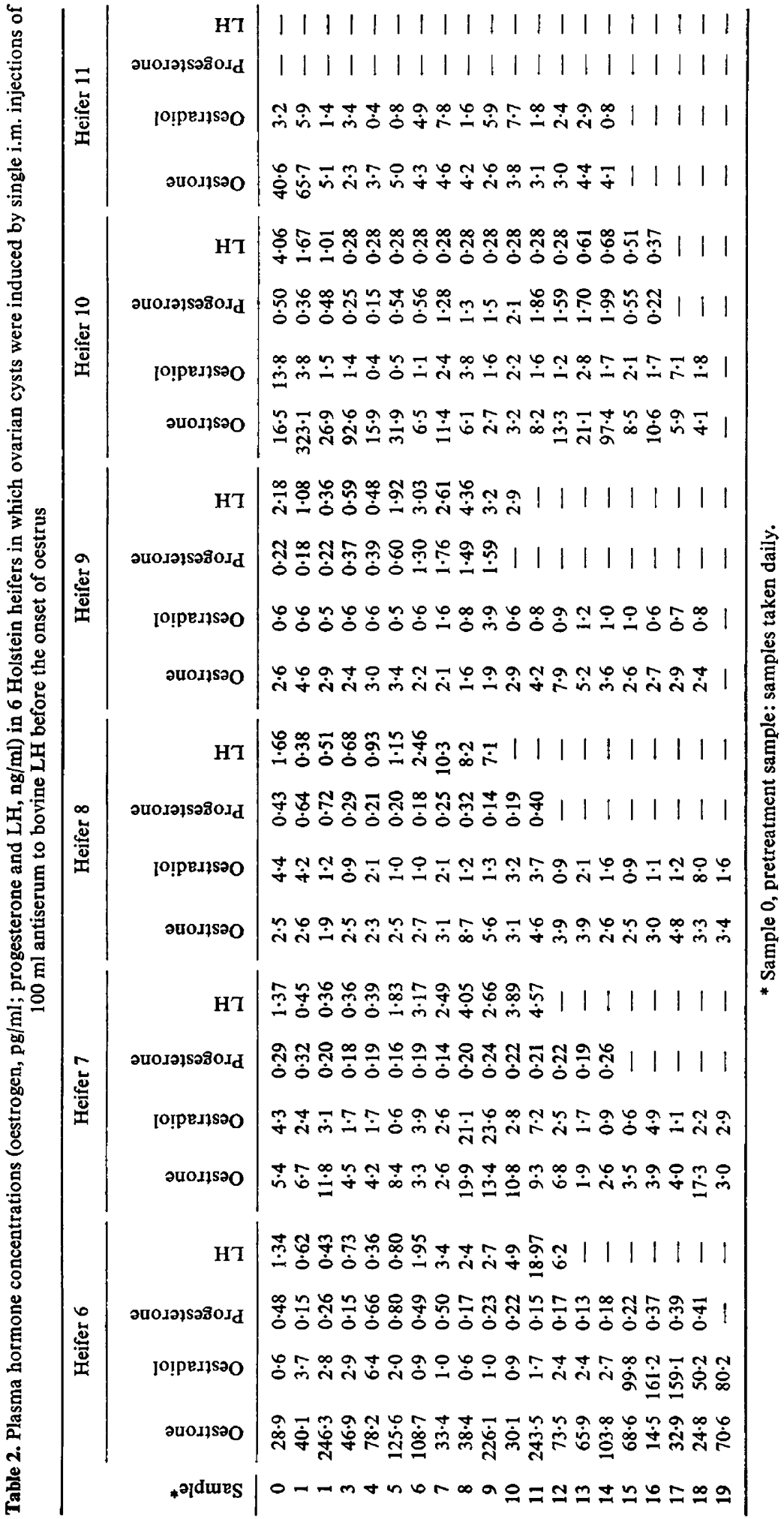


was altered for several months. The clinical findings were consistent with the hormonal responses observed. The high plasma oestradiol concentrations were associated with increased uterine tone, presence of vaginal mucus and restlessness.

\section{Experiment 2}

Oestrus. Oestrus was delayed in all 6 heifers after treatment with the LH antiserum. Oestrus occurred on Days 5, 6, 7, 10 and 10 after treatment in five of the animals giving treatment cycle lengths from 29 to 39 days, but was delayed until 62 days after treatment in Heifer 11. The first cycle after treatment varied from 9 to 42 days and the second from 18 to 35 days.

Cyst formation. Cysts were produced in all 6 heifers. The cysts were first diagnosed at 5, 7, 17, 20 and 21 days after injection and, in contrast to those formed after oestradiol treatment, were larger $(5-6 \mathrm{~cm})$ and relatively firm.

Hormonal responses. The plasma hormone concentrations are shown in Table 2. Oestradiol concentrations remained low (generally $<5 \mathrm{pg} / \mathrm{ml}$ ) for 15 days after treatment, while plasma oestrone concentrations were sporadically elevated. Elevated plasma LH concentrations appeared to precede the increase in plasma oestrogens.

Rectal palpations suggested that Heifers $6,7,8$ and 11 ovulated immediately after the antiserum injection. Plasma LH peaks may have occurred in these animals and were unobserved because of the infrequent blood sampling at this time. Functional $\mathrm{CL}$ were not formed, however, and the animals returned to oestrus 6-10 days later; they then failed to ovulate and the ovaries became cystic. The normal preovulatory LH surge was apparently blocked by the LH antiserum in Heifers 9 and 10, since neither elevated plasma $\mathbf{L H}$ concentrations nor ovulation were observed and cysts were diagnosed on Days 5 and 7 after treatment. Oestrus did not occur in Heifer 11, although ovulation did, and the follicle that then developed became cystic.

\section{Discussion}

The development of cystic ovaries after administration of $5 \mathrm{mg}$ oestradiol valerate on Day 16 of the cycle confirms the results of Wiltbank, Ingalls \& Rowden (1961). Administration of oestradiol valerate at a time of declining progesterone concentrations appeared to cause release of ovulatory amounts of $\mathrm{LH}$ similar to those released in normal animals at the onset of oestrus in Heifers 2 and 3 , as suggested by the studies of Hobson \& Hansel (1972). This response was not observed in the other 3 heifers, probably because the blood samples were taken only once every $24 \mathrm{hr}$. This premature LH release prevents ovulation of the developing follicle at the normal time, and the follicle then becomes a cyst. The increase in plasma oestradiol concentrations on Day 2 is probably due to the exogenous hormone. However, the subsequent increase seen, especially in Heifers 3 and 4, appeared to depend on the ability of the cysts to produce oestrogens. Continual high plasma concentrations of oestradiol in cows with naturally occurring cystic ovaries have been reported by Glencross \& Munro (1974) and Dobson (1973), and Lunaas, Refsdal \& Garm (1974) found a continued high oestrogen excretion in 2 cows with cystic ovaries. The fluctuations in plasma oestradiol level seen in some heifers in the present study suggest rhythmic ovarian endocrine activity. Nymphomania associated with high oestrogen production, as seen in Heifer 4, has also been reported by Dobson (1973), Glencross \& Munro (1974) and Lunaas et al. (1974).

The delayed oestrus observed in Exp. 2 when LH antiserum was administered accords with the findings of Snook et al. (1969) and Schechter (1973), who injected a similar antiserum on Days 2 and 16 respectively. After treatment on Days 16-20 of the cycle the average length of the treatment cycle was 36.7 days (Snook et al., 1969).

The neutralization effect of the anti-LH serum in the present study lasted for a shorter period (4-6 days) than the 12-14 days reported by Snook et al. (1969) and Schechter (1973). Contrary to the suggestion by Fuller \& Hansel (1970) and Snook et al. (1969), the present study also provides evidence that oestrogen secretion occurs during the effective neutralization period of the anti-LH serum,'suggesting,that FSH alone could play an important role in oestrogen secretion in the cow. 
Although cysts were produced by treatment with anti-LH serum and oestradiol valerate, the types of cyst and the oestrogen response differed. The antiserum treatment produced large hard cysts, and high plasma oestrone concentrations. Administration of oestradiol valerate produced soft small cysts and high plasma oestradiol and low plasma oestrone concentrations. Both treatments suppressed plasma progesterone for long periods, probably by interfering with the formation of functional CL. It is possible that the two treatments evoke cystic responses by different mechanisms. The difference in the consistency and size of the cysts produced by the two treatments is similar to the difference one observes in naturally occurring follicular and luteinized cysts. Further studies are needed to determine the histological changes induced by these treatments.

We are indebted to the Population Council, Biomedical Division for the award of a Fellowship to one of us (R.N.). The excellent technical assistance of Catherine Mazauskas and Raymond Saatman is gratefully acknowledged.

\section{References}

CASIDA, L. \& ChAPMAN, A. (1951) Factors affecting the incidence of cystic ovaries in a herd of Holstein cows. J. Dairy Sci. 34, 1200-1205.

Donson, H. (1973) Reproductive hormones of the non-pregnant cow. Ph.D. thesis, Liverpool University.

Donaldson, L.E. \& Hansei., W. (1968) Cystic corpora lutea and normal and cystic Graafian follicles in the cow. Aust. vet. J. 44, 304-308.

Echtrenkamp, S.E. \& Hansel, W. (1973) Concurrent changes in bovine plasma hormone levels prior to and during the first postpartum estrous cycle. $J$. Anim. Sci. 37, 1362-1370.

FulleR, G.B. \& HANSEL, W. (1970) Regression of sheep corpora lutea after treatment with anti-bovine luteinizing hormone. J. Anim. Sci. 31, 99103.

GARM, O. (1949) Study on bovine nymphomania. Acta endocr., Copenh., Suppl. 3, 1-45.

Glencross, R.G. \& Munro, I.B. (1974) Oestradiol and progesterone levels in plasma of a cow with ovarian cysts. Vet. Rec. 95, 169.

Henricson, B. (1957) Genetical and statistical investigations into so-called cystic ovaries in cattle. Acta agric. scand. 7, 1-28.

Hixon, J.E., Nadaraja, R., Schechter, R.J. \& Hansel, W. (1973) Prostaglandin $F_{2 a}$-induced stimulation of estrone and estradiol-17 $\beta$ secretion in cattle. Prostaglandins 4, 679-685.

Hobson, W.C. \& Hansfi, W. (1972) Plasma LH levels after ovariectomy, corpus luteum removal and estradiol administration in cattle. Endocrinology 91, 185-191.

LunaAs, T., Refsdal, A.O. \& Garm, O. (1974) Urinary excretion of oestrone and oestradiol-17 $\alpha$ in cows with cystic ovaries. Acta endocr., Copenh. 75, $350-356$.

McENTeE, K. (1958) Cystic corpora lutea. Int. J. Fert. 3, 120-126.

RoBerTs, S.J. (1971) Veterinary Obstetrics and Genital Diseases, 2nd edn, pp. 421-435. S. J. Roberts, Ithaca.

SCHECHTER, R.J. (1973) Control of the bovine estrous cycle. $\mathrm{Ph} . \mathrm{D}$. thesis, Cornell University.

SNOOK, R.B., BrunNer, M.A., SaAtman, R.R. \& HANSEL, W. (1969) The effect of antisera to bovine LH in hysterectomized and intact heifers. Biol. Reprod. 1, 49-58.

WILTBANK, J.N. (1966) Modification of ovarian activity in the bovine following injection of oestrogen and gonadotrophin. J. Reprod. Fert., Suppl. 1, 1-10.

Wiltbank, J.N., Ingalls, J.E. \& Rowden, W.W. (1961) Effects of various forms and levels of oestrogens alone or in combination with gonadotrophins on the estrous cycle of beef heifers. J. Anim. Sci. 20, 341-346.

Yamauchi, M., Ashida, K. \& INUI, S. (1954) Studies on the ovarian cyst in the cow. III. Endocrinological studies on the cause of the ovarian cyst. Jap. J. vet. Sci. 16, 65-71. 